\title{
A NOVEL AND FAST CORNER DETECTION METHOD FOR SAR IMAGERY
}

\author{
Niangang Jiao ${ }^{\mathrm{ab}}$, Wenchao Kang ${ }^{\mathrm{ab}}$, Yuming Xiang ${ }^{\mathrm{ab} *}$,Hongjian You ${ }^{\mathrm{ab}}$ \\ ${ }^{a}$ Key Laboratory of Technology in Geo-spatial Information Processing and Application System, Institute of Electronics, \\ Chinese Academy of Sciences, Beijing, China \\ jiaoniangang16@mails.ucas.ac.cn,xshzhdm@163.com, z199208081010@163.com, hjyou@mail.ie.ac.cn \\ ${ }^{\mathrm{b}}$ University of Chinese Academy of Sciences, Beijing, China
}

\section{Commission I, WG I/3}

KEY WORDS: SAR; Corner detection; FAST; Ratio.

\begin{abstract}
:
Corners play an important role on image processing, while it is difficult to detect reliable and repeatable corners in SAR images due to the complex property of SAR sensors. In this paper, we propose a fast and novel corner detection method for SAR imagery. First, a local processing window is constructed for each point. We use the local mean of a $3 \times 3$ mask to represent a single point, which is weighted by a Gaussian template. Then the candidate point is compared with 16 surrounding points in the processing window. Considering the multiplicative property of speckle noise, the similarity measure between the center point and the surrounding points is calculated by the ratio of their local means. If there exist more than $\mathrm{M}$ continuous points are different from the center point, then the candidate point is labelled as a corner point. Finally, a selection strategy is implemented by ranking the corner score and employing the non-maxima suppression method. Extreme situations such as isolated bright points are also removed. Experimental results on both simulated and real-world SAR images show that the proposed detector has a high repeatability and a low localization error, compared with other state-of-the-art detectors.
\end{abstract}

\section{INTRODUCTION}

With the rapid development of modern radar sensors, the amount of high resolution radar images is increasing quickly both for optical images and Synthetic Aperture Radar (SAR) images. Compared with optical images, SAR images are more stable owing to its property of all-time and all-weather. Due to the impact of coherent imaging mechanism and side looking geometry, SAR images are always corrupted by massive coherent speckles. In SAR image processing systems, corner detection plays an important role. Corner points that are invariant to rotation and translation are important low-level features, which have been widely used in image registration, change detection and object extraction (LIU Yan, 2017). Lots of research efforts have been devoted to this topic, such as Harris detector (Harris and Stephens, 1988), Hessian detector (Bay et al., 2006), SUSAN detector (MA et al., 2007), FAST detector (Rosten and Drummond, 2006).

However, typical algorithms including FAST and Harris detectors are not suitable to SAR image due to the speckle noise. Recently, some modifications of these corner detectors have been proposed. Replacing the original gradient with the logarithmic ratio of the exponentially weighted averages (ROEWA) operator, Dellinger proposed a multi-scale SAR-Harris detector (Dellinger et al., 2015). Yan proposed a SAR-FAST detector based on FAST (LIU Yan, 2017). The SAR-FAST method firstly employed rolling guidance filter to suppress speckle noise, then corner points were determined by quantitative analysis of the dissimilarities of the detection windows on the extended circle and the center window. However, these algorithms still have some limitations. The computation cost of multi-scale SAR-Harris detector is high when considering the real-time applications (Wu et al., 2016). For the SAR-FAST detector, its performance heavily depends on the fil-

\footnotetext{
*Corresponding author
}

tered image, while the filtering process (Zhang et al., 2014) always has a high computational complexity and may lose some important information.

\section{RELATED WORK}

\subsection{Harris Method}

Harris and Stephens proposed a corner detection algorithm known as Harris algorithm (Harris and Stephens, 1988). This corner detector improves the Moravec corner detector using the local auto-correlation function. The local autocorrelation function is given by:

$$
E_{x, y}=\sum_{u, v} w_{u, v}\left[I_{x+u, y+v}-I_{u, v}\right]^{2}
$$

where $\mathrm{I}$ is the image intensity, $(x, y)$ is the small shift, $w$ denotes a circular Gaussian template to suppress the noise. Then the function $E$ can be approximated by two first gradients on both $x$ and $y$ directions:

$$
\begin{aligned}
& E(x, y)=(x, y) M(x, y)^{T} \\
&=(x, y)\left[\begin{array}{cc}
(\partial I / \partial x)^{2} & (\partial I / \partial x)(\partial I / \partial y) \\
(\partial I / \partial x)(\partial I / \partial y) & (\partial I / \partial y)^{2}
\end{array}\right] \otimes w
\end{aligned}
$$

where $M$ is the local covariance matrix which has two eigenvalues $\lambda_{1}, \lambda_{2}$.

$\lambda_{1}$ and $\lambda_{2}$ are proportional to the curve of local self-related functions. They have three cases: If $\lambda_{1}$ and $\lambda_{2}$ are both small, the intensity in the local processing window is of approximately constant, then the local autocorrelation function is flat. If one of them is small and the other one is large, the window straddles an 
edge. If both of them are large, this indicates a region containing a corner. To finally find the location of a corner pixel, a corner response function is calculated:

$$
R=D E T(M)-k T r(M)^{2}
$$

where $\operatorname{Det}(M)$ and $\operatorname{Tr}(M)$ represent determinant and trace of matrix $M$ respectively. Then the corner pixel can be extracted by finding the local max response in a local window.

\subsection{FAST Method}

Liu et al. improved the original FAST corner detection algorith$\mathrm{m}$ to make it suitable for SAR image (LIU Yan, 2017). Due to speckle noise of radar images, the SAR-FAST method first employed the rolling guidance filter (Zhang et al., 2014) to suppress the noise. In FAST detector (Rosten and Drummond, 2006), a local processing window is constructed for each pixel. By comparing the center point with the surrounding points, the corners can be detected. The average intensity of the center pixel $P$ is compared with the $j$ th points $Q_{j}$. If

$$
\left|P-Q_{j}\right| \geq T h
$$

then the $j$ th point is different from the center point, where $T h$ is a fixed threshold value. If there exist more than $M$ continuous points that are different from the center poitn, then the center pixel can be detected as a corner.

\section{METHODOLOGY}

Figure 1 illustrates the flow chart of the proposed corner detector. The whole procedure mainly consists of three parts: local processing window construction, candidate points detection by similarity and false points elimination.

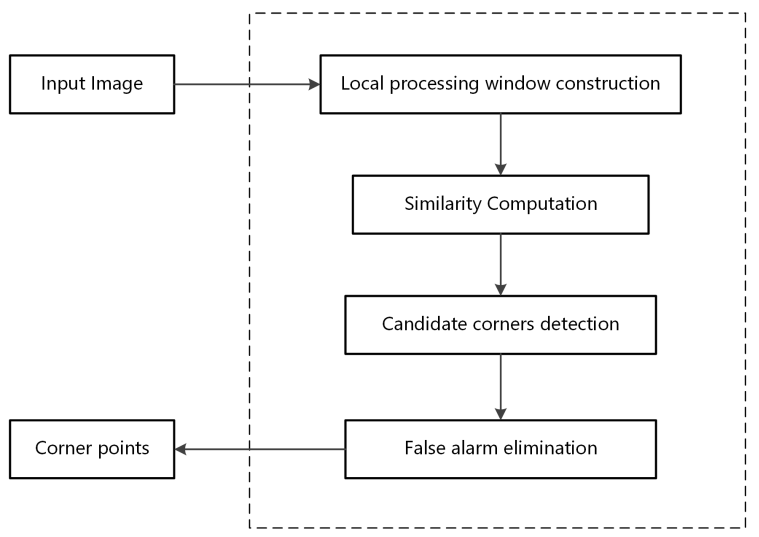

Figure 1. Flow chart of the proposed detector

First, inspired by the efficient FAST detector used in optical image corner detection (Rosten and Drummond, 2006), we construct a local processing window for each pixel, which is shown in Figure 2. As mentioned in Section 1, SAR images are strongly corrupted with the speckle noise. Considering the effect of noise, we utilize a 3x3 Gaussian template (Babaud et al., 1986) to represent each single pixel, then the size of processing window is 19x19. A Gaussian template can suppress the noise to some extent. Moreover, local structural information has been taken into consideration in each template, the adjacent pixels can help us identify the candidate pixel more efficiently (Wei and Feng, 2015). Consequently, the proposed method is more stable and more robust to speckle noise.

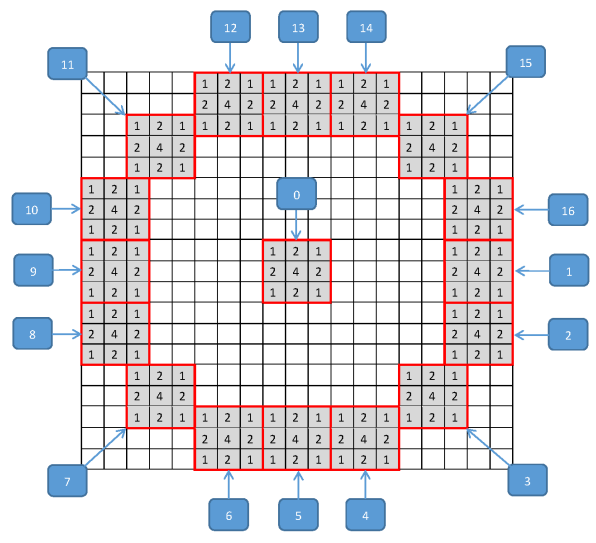

Figure 2. The local processing window and Gaussian templates.

Second, the similarity measure used in the FAST detector is the intensity difference, while the speckle noise is generally modelled as a multiplicative noise (Touzi et al., 1988). Hence, the intensity difference may lead to many false alarms, especially in the homogenous areas. Here, we replace the intensity difference with the ratio difference in the detector . Benefiting from the property of constant false alarm rate (CFAR) for SAR imagery, ratio-based detectors have been widely used in edge detection and descriptor extraction, which are robust to the speckle noise (Xiang et al., 2017, Shui and Cheng, 2012). The proposed ratio difference is calculated as follows:

$$
\operatorname{Ratio}(k)=\frac{\mu(0)}{\mu(k)}, k=1,2, \ldots, 16
$$

where $\mu(0)$ represents the average of the center template 0 , and $\mu(k)$ denotes the average of the template $\mathrm{k}$. Based on the processing window, 16 templates around the center template are utilized to calculate the ratio difference. Accordingly, we can obtain 16 ratio differences to measure the structure of the candidate pixel. For each ratio value, the similarity measure is derived by comparing it with a threshold:

$$
d_{k}=\left\{\begin{array}{ll}
0, & \text { if } 1 / \text { Th }<\text { Ratio }(k)<T h \\
1, & \text { if Ratio }(k)<T h \\
2, & \text { if Ratio }(k)>1 / T h
\end{array}\right\}
$$

where $T h$ is a threshold. We consider the candidate pixel is a candidate corner point if more than a half templates are continuously larger or smaller than the center template. Instead of comparing all the templates one by one, we employ an acceleration method in the detection procedure. We first compare the 1st and 9th templates with the center template, if the two templates are similar to the center template $d_{1}=0$ and $d_{9}=0$, then the candidate pixel is not a corner. Then we test the 5th and 13th templates. After testing all the diagonal templates, we can operate the full 16templates test on the remaining candidate pixels by accumulating the sum of $d_{k}$.

After the detection process, many candidate corners are selected, while there exist some false detections and duplicate detections. In order to solve this issue, a false point elimination method is employed. First, we assign a score function to each candidate corners based on its $d_{k}$ values. For a candidate corner, the more continuous nonzero values, the higher its score. Then a non-maxima suppression is utilized to select the best corner point. 


\section{EXPERIMENTAL RESULTS}

In this section, the proposed detector is compared with two stateof-the-art methods, SAR-FAST detector and SAR-Harris detector. We first evaluate these detectors on several simulated SAR images, then a real-world SAR image is tested. For the simulated SAR images, two quantitative metrics are used to evaluate the performance, localization error and number of correctly-detected points. For satellite SAR images, quantitative comparisons are difficult because the ground truth (GT) maps do not exist for realworld images. We compare the visual effects among these detectors. For fairness, all of the detectors use the same pre-processing and post-processing method. Since we want to test the robustness of these detectors versus strong speckle noise and the filtering process is always time-consuming, we do not apply a filter to smooth the raw image. The parameters of SAR-FAST detector and SAR-Harris detector follow their authors instructions. For the proposed detector, we set the threshold Th as 0.7 for images with high level of noise, while 0.8 for images with low levels of noise and 0.6 for the real-world SAR image.

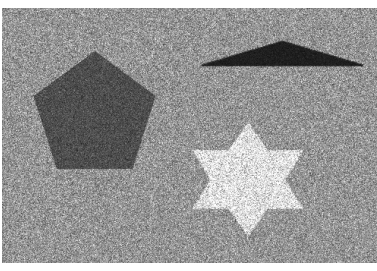

(a)

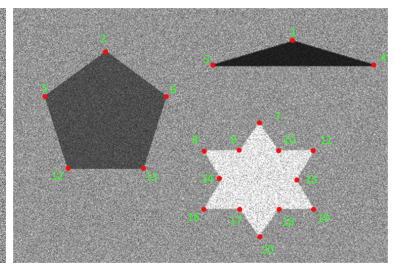

(b)
Figure 3. (a) The simulated three-look SAR image. (b) The ground truth map.

\subsection{Comparison on Simulated SAR Images}

In this experiment, a polygon image with simulated three-look speckle noise is used, which is shown in Figure 3(a). Figure 3(b) shows the ground truth corners of this simulated image. The two quantitative measure criteria are localization error and number of correctly-detected points. We consider a candidate point is a correctly-detect point, if its coordinate satisfies:

$$
\sqrt{\left(x-x_{o}\right)^{2}+\left(y-y_{o}\right)^{2}}<d
$$

where $\left(x_{o}, y_{o}\right)$ denotes the coordinate of corresponding ground truth corner, $d$ is a distance thredhold. The localization error is calculated by the distances of all the correcly-detected corners. The larger the number of correcly-detected points, the better the detctor, accordingly, the smaller the localization error, the better the detector. In order to evaluate the detection performance, we first compare these detectors on the three-look simulated SAR image. Figure 4 shows the detection results of all the three detectors on the simulated SAR image. It can be observed that the proposed detector extracts all the correct corners, while the other two methods miss some corners.

In order to further analysis the performance, we compare these detectors on simulated images of different noise levels. High number of looks denotes low noise level, while small number of looks represents strong noise level. Figure 5(a) shows the localization errors versus the noise level and Figure 5(b) illustrates the number of correcly detected points versus the noise level.

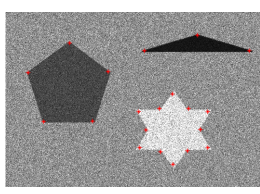

(a)

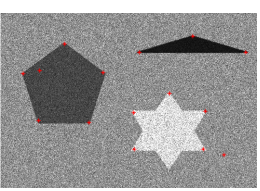

(b)

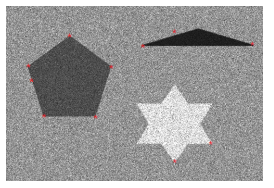

(c)
Figure 4. The detection results of three detectors on the simulated SAR image. (a) Proposed. (b) SAR-FAST. (c) SAR-Harris.

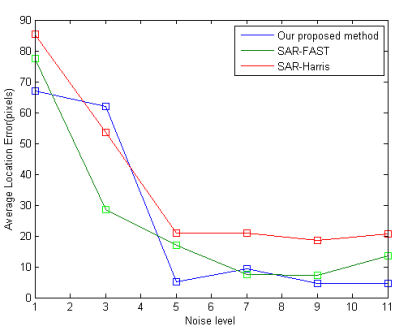

(a)

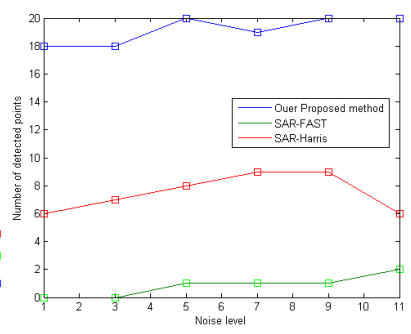

(b)
Figure 5. The performence of three detectors on simulated SAR images of different noise levels. (a) The localization errors versus the noise level. (b) The number of correcly detected points versus the noise level.

\subsection{Comparison on a Real-world SAR Image}

Finally, a satellite image is tested in this experiment. The image was taken from a GaoFen-3 satellite with Ultra Fine Stripmap mode and $\mathrm{HH}$ polarization, its an amplitude image with a size of $800 \times 500$. The detection results of three comparative detectors are shown in Figure 6. We use the same parameters for the SARFast and SAR-Harris detectors. For the proposed detector, we set the threshold Th to 0.6. It can be observed from the detection results that the proposed detector extract reliable corners both in the high contrast regions and low contrast regions.

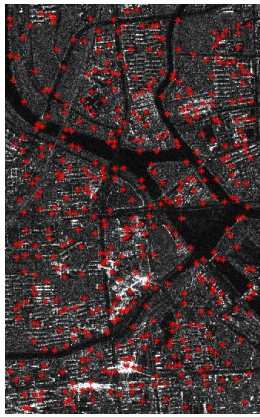

(a)

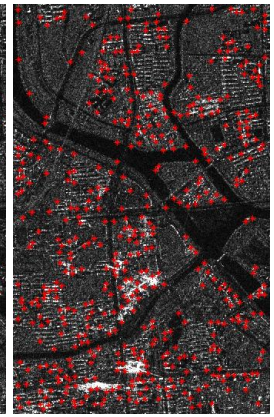

(b)

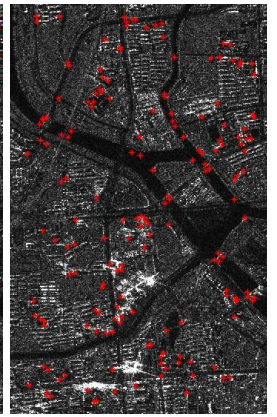

(c)
Figure 6. The detection results of three detectors on the real-world SAR image. (a) The result of our proposed method.

(b) The result of SAR-FAST method. (c) The result of SAR-Harris method.

\section{CONCLUSION}

In this paper, a novel and fast corner detection method for SAR imagery is proposed. By replacing the intensity difference and constructing a new processing window, the original FAST algorithm is improved to be adapted to SAR imagery. Considering 
the effect of speckle noise, we utilize a Gaussian template to represent one pixel, and the ratio-based similarity measure is proposed to detect the candidate corner. In addition, a false alarm elimination strategy is employed based on a score function and non-maxima suppression. Experimental results demonstrate that the proposed corner detector gives a good performance with high repeatability and low localization error.

\section{REFERENCES}

Babaud, J., Witkin, A. P., Baudin, M. and Duda, R. O., 1986. Uniqueness of the gaussian kernel for scale-space filtering. IEEE transactions on pattern analysis and machine intelligence (1), pp. 26-33.

Bay, H., Tuytelaars, T. and Van Gool, L., 2006. Surf: Speeded up robust features. Computer vision-ECCV 2006 pp. 404-417.

Dellinger, F., Delon, J., Gousseau, Y., Michel, J. and Tupin, F., 2015. Sar-sift: a sift-like algorithm for sar images. IEEE Transactions on Geoscience and Remote Sensing 53(1), pp. 453-466.

Harris, C. G. and Stephens, M. J., 1988. A combined corner and edge detector. In: Alvey Vision Conference, pp. 147-151.

LIU Yan, YU Huai, Y. W. L. L., 2017. Sar image registration using sar-fast corner detection. Journal of Electronics and Information Technology 39(2), pp. 430-436.

MA, G., FANG, Z. and YAO, Z., 2007. Performance analysis and comparison of susan edge detector [j]. Modern Electronics Technique 8, pp. 189-191.

Rosten, E. and Drummond, T., 2006. Machine learning for highspeed corner detection. Computer vision-ECCV 2006 pp. 430443.

Shui, P.-L. and Cheng, D., 2012. Edge detector of sar images using gaussian-gamma-shaped bi-windows. IEEE Geoscience and Remote Sensing Letters 9(5), pp. 846-850.

Touzi, R., Lopes, A. and Bousquet, P., 1988. A statistical and geometrical edge detector for sar images. IEEE Transactions on geoscience and remote sensing 26(6), pp. 764-773.

Wei, Q.-R. and Feng, D.-Z., 2015. An efficient sar edge detector with a lower false positive rate. International Journal of Remote Sensing 36(14), pp. 3773-3797.

Wu, B., Zhou, S. and Ji, K., 2016. A novel method of corner detector for sar images based on bilateral filter. In: Geoscience and Remote Sensing Symposium (IGARSS), 2016 IEEE International, IEEE, pp. 2734-2737.

Xiang, Y., Wang, F., Wan, L. and You, H., 2017. Sar-pc: Edge detection in sar images via an advanced phase congruency model. Remote Sensing 9(3), pp. 209.

Zhang, Q., Shen, X., Xu, L. and Jia, J., 2014. Rolling guidance filter. In: European Conference on Computer Vision, Springer, pp. $815-830$. 